patients, and complete control was achieved in $25(42 \%)$. Adverse events occurred in 10 $(16.7 \%)$ and included drowsiness, aggressive behavior, ataxia, dizziness, diplopia, and leg cramps. None had hyponatremia or skin rash. Of 24 patients switched from carbamazepine to oxcarbazepine because of toxic effects or poor seizure control, $79 \%$ had $>50 \%$ seizure reduction, and $37.5 \%$ became seizure-free. Serum levels were not determined. (Kothare SV, Khurana DS, Mostofi $\mathrm{N}$ et al. Oxcarbazepine monotherapy in children and adolescents: a single-center clinical experience. Pediatr Neurol Oct 2006;35:235-239). (Respond: Dr Kothare, St Christopher's Hospital for Children, Division of Pediatric Neurology; Director, Pediatric Epilepsy Program, Erie Avenue at Front Street, $3^{\text {rd }}$ floor ACP, Suite 3314, Philadelphia, PA 19134).

COMMENT. Oxcarbazepine monotherapy, a keto analog of carbamazepine, is effective and without serious side effect in children and adolescents with partial epilepsy. It is approved as monotherapy in children $>4$ years old and as adjunctive therapy in children $>2$ years old (in Europe, for $>6$ year-olds). The inverse relation between dose and age is a reflection of a more rapid clearance of oxcarbazepine in younger patients.

\title{
VALPROATE-RELATED HYPERINSULINEMIA
}

Changes in insulin metabolism during treatment with valproate (VPA) were investigated in a study of 51 patients receiving monotherapy ( 31 male and 20 female) and 45 healthy controls at University of Oulu, and Hospital of Children and Adolescents, University of Helsinki, Finland. VPA-treated patients had fasting hyperinsulinemia, while the fasting serum proinsulin and C-peptide levels did not differ from controls. Proinsulin/insulin and Cpeptide/insulin ratios were reduced in VPA-treated patients, and fasting plasma glucose levels were lower compared to controls. These changes were independent of the sex, seizure type, weight gain, and pretreatment weight, and were not due to weight gain or obesity. VPA does not induce insulin secretion but interferes with insulin metabolism in the liver, resulting in hyperinsulinemia and reduced plasma glucose. The obesity induced by VPA is secondary to the increase in circulating insulin. (Pylvanen V, Pakarinen A, Knip M, Isojarvi J. Characterization of insulin secretion in valproate-treated patients with epilepsy. Epilepsia Sept 2006;47:1460-1464). (Reprints: Dr V Pylvanen, Department of Neurology, PO Box 5000, 90014 Oulu, Finland).

COMMENT. VPA causes hyperinsulinemia and consequent fall in plasma glucose by interference with insulin metabolism in the liver. The hyperinsulinemia may then induce increased weight gain.

Serum total amylase, pancreatic amylase and lipase in VPA-treated epilepsy children. In a study of 23 patients, serum pancreatic amylase was decreased at 6 and 12 months treatment with VPA, whereas serum total amylase and lipase were unchanged. Nonpancreatic isoenzyme activities of amylase were higher at 6 or 12 months. Nonpancreatic amylase, probably derived from salivary glands, may be increased in children treated with VPA, and is not a reason to interrupt therapy. Measurement of serum pancreatic amylase and/or lipase is indicated when total amylase is increased without clinical symptoms of pancreatitis. (Voudris K et al. Brain Dev Oct 2006;28:572-575). 